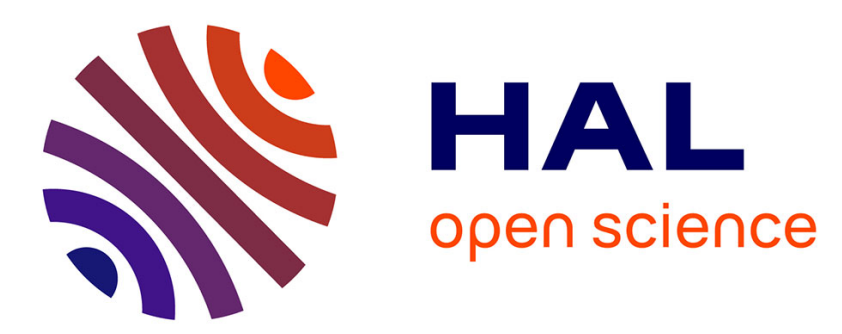

\title{
Working principle description of the wireless passive EM transduction pressure sensor
}

\author{
M.M. Jatlaoui, F. Chebila, Patrick Pons, Hervé Aubert
}

\section{To cite this version:}

M.M. Jatlaoui, F. Chebila, Patrick Pons, Hervé Aubert. Working principle description of the wireless passive EM transduction pressure sensor. European Physical Journal: Applied Physics, 2011, 56 (1), pp.13702. 10.1051/epjap/2011100220 . hal-00736280

\section{HAL Id: hal-00736280 \\ https://hal.science/hal-00736280}

Submitted on 28 Sep 2012

HAL is a multi-disciplinary open access archive for the deposit and dissemination of scientific research documents, whether they are published or not. The documents may come from teaching and research institutions in France or abroad, or from public or private research centers.
L'archive ouverte pluridisciplinaire HAL, est destinée au dépôt et à la diffusion de documents scientifiques de niveau recherche, publiés ou non, émanant des établissements d'enseignement et de recherche français ou étrangers, des laboratoires publics ou privés. 


\title{
Working Principle Description of the Wireless Passive EM
}

\section{Transduction Pressure Sensor}

Mohamed Mehdi Jatlaoui ${ }^{1}$, Franck Chebila ${ }^{1,2}$, Patrick Pons ${ }^{1}$ and Herve Aubert ${ }^{1,2}$ a

1 CNRS; LAAS; 7, avenue colonel Roche, F-31077 Toulouse, France

2 University of Toulouse; UPS; INSA; INP; ISAE; LAAS; F-31077, Toulouse, France

Received: date / Revised version: date

\begin{abstract}
The development of a new passive wireless pressure sensor, based on an electromagnetic transduction approach, is reported. The sensing element is a flexible high resistivity silicon membrane located above a coplanar quarter-wavelength resonator. The comprehensive coverage of the physical bases is beyond the scope of this paper. For the remote extraction of the applied pressure value, the passive pressure sensor is connected to a broadband horn antenna via a coaxial delay line. When interrogated by a frequency modulated continuous wave radar, the level of the backscattered signal changes versus the pressure applied to the proof body. Through this interrogation principle, the sensor provides load impedance that is reflected back to the radar reader: the measured dynamic is about $0.8 \mathrm{dBm} /$ Bar. This completely passive and wireless pressure telemetry micro-sensor has been designed, fabricated and characterized, thereby eliminating the need for contact, signal processing circuits, and power supplies needed by conventional active sensors.
\end{abstract}

\section{Introduction}

Today, more and more growing demands of the industry for implementation of completely passive sensor system are observed. In fact, in many rising applications a wired connection between the sensor and the data processing system cannot be installed and simplicity of the sensor is Send offprint requests to:

a Present address:jatlaoui@laas.fr desirable. There is a need for wireless telemetry system that requires no physical contact with the sensor and no active elements such as power supplies, signal processing circuits, or batteries. In the past, several systems consisting of an active battery-powered sensor unit have been presented $[1,2]$. The major problem of these active-based systems is the necessary energy source, which limits the lifetime, performance, cost and may add significant weight 
and volume to the sensor unit. Other passive systems have been also developed such as Radio Frequency IDentification (RFID) tags and Surface Acoustic Wave (SAW) based sensors $[3,4]$. But passive RFID tags present the disadvantage of short reading range while the main disadvantages of acoustic sensors are sensitivity to contamination, difficulty of calibration, and the need for optimized signal processing algorithm [5].Moreover, due to the fact that the RF interrogation signal has to cover twice the distance between the transceiver and the sensor unit without amplification, the attenuation is doubled compared to conventional battery-powered sensor systems [6]. Thus, the signal amplitude received by the transceiver unit is many orders lower than the amplitude of the transmitted interrogation signal. In this paper, the development of a new passive and wireless pressure sensor, based on an electromagnetic transduction approach, is reported. The sensor design covers different disciplines such as microwaves, materials, micro-fabrication, and mechanics. The comprehensive coverage of the physical bases is beyond the scope of this paper and new measurement results dealing with the wireless interrogation technique of the pressure sensor unit are presented. The sensor cell can be considered as a passive scatterer having a reflectivity (or radar cross section) that varies with -or is controlled by- the applied pressure. These measurements show that the proposed wireless passive system (composed of: sensor, antenna and radar) is functional and can be used for remote measurements of the applied pressure. The paper is structured as follows. In Section 2 the design approach of the pressure micro-sensor is reported. In Section 3, the detailed working principle is described. Measurement results are shown and discussed in Section 4. Finally conclusions and perspectives of this research work are presented in Section 5.

\section{The EM pressure Sensor}

\subsection{The Concept}

Pressure sensors generally consist of a flexible mounted diaphragm which undergoes a deflection due to different pressure between both sides. Recently we have presented new pressure sensor based on an Electromagnetic (EM) transduction approach $[7,9]$. The principle of the sensor is based on the change of an electromagnetic field distribution in the interface between a planar resonator and a membrane placed above. In this region, the EM field is evanescent and consequently, a small deflection of the membrane due to the applied pressure modifies significantly the EM field distribution: a shift in the microwave resonant frequency of the resonator occurs and from this shift estimation of the applied pressure may be derived. To fabricate a high-sensitivity sensor, based on this principle we must achieve a high mechanical/electromagnetic coupling effect for very small membrane displacement (few micrometers). We settled an operating (resonant) frequency of $\mathrm{f} 0=30 \mathrm{GHz}$. At this frequency, the dimensions of microwave circuits are about few millimeters. So we can intend to realize rather compact pressure sensors for various applications (automotive, aeronautical). It is necessary to note also that we are in perfect adequacy with frequen- 
cies ranges used for Radar system: Frequency Modulated Continuous Wave (FMCW) Radar can remotely interrogate the passive pressure sensor at a range of some tens of meters. The sensor unit is then viewed as a passive scatterer having radar cross section that varies with the applied pressure.

\subsection{Sensor Structure}

As shown in figure 1, the pressure sensor is composed of four parts:

*The membrane: this important element on which the pressure is applied is fabricated using high resistivity silicon $(>3 \mathrm{k} \Omega . \mathrm{cm})$. This material has a dielectric permittivity of 11,6 and is chosen due to its well known mechanical properties as well as the fabrication process. The geometrical shape of the membrane is given by the geometry of the cavity where the planar resonator is deposited. The silicon membrane will be sealed to Pyrex by an anodic bonding;

* The substrate is in Pyrex of type borosilicate (CORNING 7740). This material presents weak RF losses with a dielectric permittivity of 4,6 . This material allows an easy assembly with the silicon. Its dilation coefficient is more or less the same of that of the silicon membrane. The Pyrex is resistant to thermal shocks, resisting to the acid baths and can be used for high temperature applications;

* The resonator consists of a quarter-wave coupled line coplanar filter (see Section 2.3). The ideal conductive material remains the gold considering its weak electric resistivity which tends to minimize the effect of the skin depth.
The gold is also characterized by its excellent resistance to chemical baths. However, in our case, we cannot use this kind of metal because in the Pyrex/Silicon bonding step, we rise in temperature up to $370^{\circ} \mathrm{C}$ and thus problems of gold diffusion may take place. Our choice settles on another metal: Aluminum $(\mathrm{Al})$, which is resistant to the chemical baths as well as gold. This metal presents weak resistive losses. The aluminium thickness is fixed to 1 micron;

* The air gap is the thin layer between the resonator and the silicon membrane placed above. In this gap the EM field is evanescent.

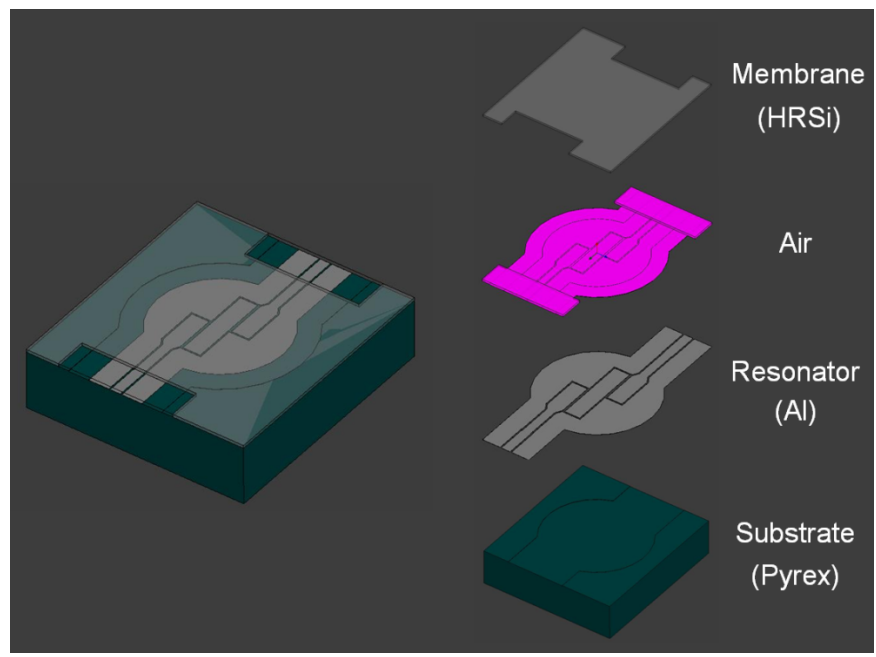

Fig. 1. EM sensor structure and its four constitutive parts (on the right side on the figure)

\subsection{Sensor Design and Dimensions}

The resonator is realized in coplanar technology. A coplanar structure presents the advantage to have an EM field distribution which is in adequacy with the EM transduction approach and requires planar pads (the ground and 
the signal line are in the same plane). Moreover, in comparison with the other transmission line technologies, the coplanar one presents more design flexibility: for the same impedance value, one can have several possible dimensions. Coplanar transmission lines offer a better compliance to perform RF measurements.

The resonator is composed of a quarter-wave coupled line filter operating at f0 $=30 \mathrm{GHz}$. The quarter-wave coupled line filter can present the same performances (bandwidth, selectivity, quality factor) as an interdigitated filter but it will be in spite of the compactness. We adopt here a structure based on a filter with only one resonant element to enhance the interaction of the silicon membrane with the evanescent EM field confined above the coplanar resonator. The Sensor topology is shown in figure 2. The parameters $\mathrm{W}, \mathrm{G}, \mathrm{S}, \mathrm{L}$ and $\lambda \mathrm{g}$ represent respectively the coplanar line width, the coplanar line gap, the coplanar coupled line gap, the central line length and the guided wavelength of the fundamental mode in the coplanar line at the operating frequency f0.

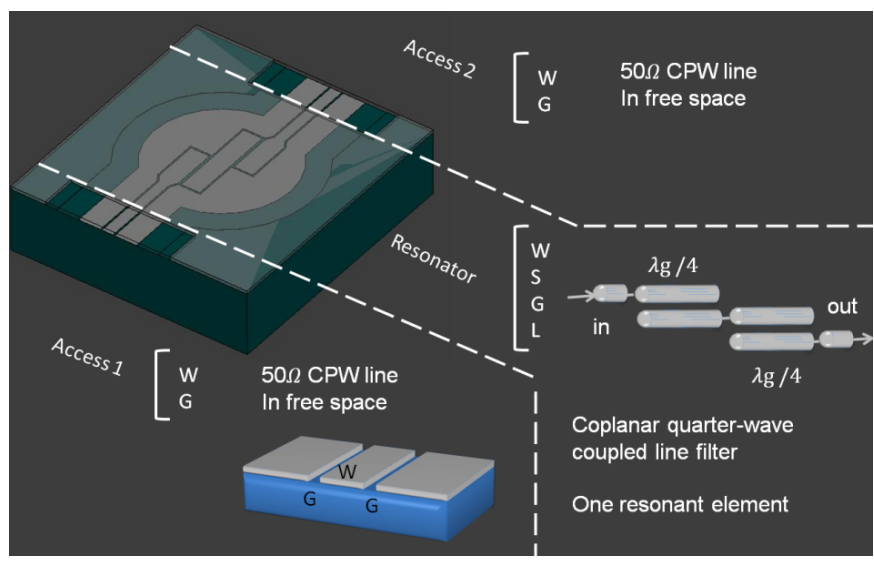

Fig. 2. EM sensor topology.CPW stands for CoPlanarWaveguide (See text for the definition of $\mathrm{W}, \mathrm{G}, \mathrm{S}, \mathrm{L}$ and $\lambda \mathrm{g}$ ).
The design of the millimeter-wave coplanar resonator is done according to the classical filter design theory $[10$, 11]. The coplanar quarter-wave coupled line resonator is designed to have a resonant frequency $\mathrm{f} 0=30 \mathrm{GHz}$ for a membrane thickness $\mathrm{h} 3=400$ microns and $\mathrm{h} 2=3$ microns where $\mathrm{h} 2$ designates the thickness of the air gap. For such resonant frequency the resonator dimensions (in microns) are given in figure 3 .

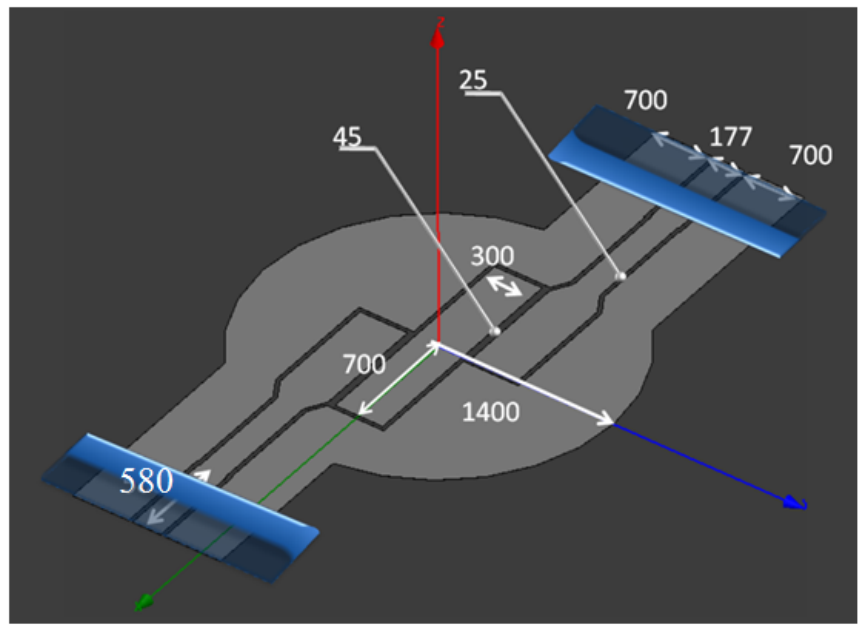

Fig. 3. Dimensions (in micrometer) of the millimeter-wave parallel-coupled half-wavelength resonator in coplanar technology

\section{How does the EM Transduction Pressure}

\section{Sensor Work?}

This section aims at (1) bringing comprehension elements of the physical phenomenon related to the electromagnetic transduction and (2) verifying this principle without a complicated design step. The working principle is described by means of an analytical model by considering a 
dielectric membrane getting closer to a coplanar waveguide transmission line with a uniform movement. This study is based on an integral and variational electromagnetic modeling theory called the Transverse Resonance Method [12].It allows the extraction of key parameters such as: the effective relative permittivity of the waveguiding structure, the sensor fundamental resonant frequency and the EM field distribution. Figure 4 shows a crosssectional view of the coplanar waveguide under study. The boundary conditions are unchanged by translation along the longitudinal Oz-axis. For simplicity reasons in the theoretical developments, the dispersion equation is derived for a coplanar line inserted in artificial rectangular metallic waveguide. This waveguide introduces boundary conditions that are not present in the original structure shown in Fig. 2. However, since the electromagnetic energy of the fundamental mode in the coplanar line is known to be very small in regions close to these boundaries, the artificially inserted metallic waveguide does not significantly participate in the dispersion phenomena while allows simplifying the theoretical developments. Moreover the metallic and dielectric losses are neglected in this simple electromagnetic modeling. The coplanar line is located on the discontinuity plane $\mathrm{xOz}$ : it consists of a metallic surface with two infinite parallel longitudinal slots. The high resistivity silicon membrane with thickness h3 and relative permittivity $\epsilon \mathrm{r} 3$ is placed above the coplanar line. Between the coplanar line and the membrane, we have a thin air gap region of thickness $\mathrm{h} 2$ and relative permittivity $\epsilon \mathrm{r} 2$ :in this region the EM field is evanescent at the operating frequency. The dielectric (Pyrex) substrate of thickness h1 on which the coplanar line is micro-machined is characterized by a relative permittivity $\epsilon \mathrm{r} 1$ and thickness $\mathrm{h} 1$.

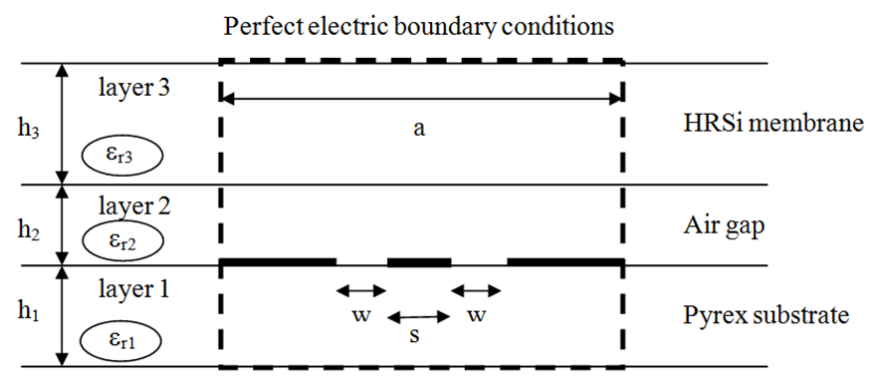

Fig. 4. Cross sectional view of the coplanar line for the theoretical developments

A good approximation of the guided wavenumber $\beta$ of the fundamental mode in this coplanar waveguiding structure is solution of the following dispersion equation (see, e.g., $[13,14]$ for details on the Transverse Resonance Method applied to planar waveguides):

$$
\begin{aligned}
& 0=\sum_{n=1,3,5, \ldots} \frac{\beta^{2}}{\left(n \frac{\pi}{a}\right)^{2}+\beta^{2}} \sin ^{2}\left(n \frac{\pi}{2} \frac{s+w}{a}\right)\left(\frac{\sin n \frac{\pi}{2} \frac{w}{a}}{n \frac{\pi}{2} \frac{w}{a}}\right)^{2}\left[Y_{n}^{T E}+\right. \\
& \left.Y_{n}^{\prime T E}+\left(\frac{n \frac{\pi}{a}}{\beta}\right)^{2}\left(Y_{n}^{T M}+Y_{n}^{\prime T M}\right)\right]
\end{aligned}
$$

where :

$$
Y_{n}^{\alpha}=Y_{M, 1 n}^{\alpha} \operatorname{coth}\left[\rho_{1 n} h_{1}\right]
$$

and

$Y_{n}^{\prime \alpha}=Y_{M, 2 n}^{\alpha} \frac{Y_{M, 3 n}^{\alpha} \operatorname{coth}\left[p_{3 n} h_{3}\right]+Y_{M, 2^{n}}^{\alpha} \operatorname{th}\left[p_{2 n} h_{2}\right]}{Y_{M, 2^{n}}^{\alpha}+Y_{M, 3 n}^{\alpha} \operatorname{coth}\left[p_{3 n} h_{3}\right] \operatorname{th}\left[p_{2 n} h_{2}\right]}$

with $\alpha=\mathrm{TE}$, TM and, for $\mathrm{i}=1,2,3$ :

$$
Y_{M, i n}^{T E}=\frac{p_{i n}}{j \omega \mu_{o}}
$$




$$
Y_{M, i n}^{T M}=\frac{j \omega \varepsilon_{o} \varepsilon_{r i}}{p_{i n}}
$$

Where

$$
p_{i n}^{2}=\left(n \frac{\pi}{a}\right)^{2}+\beta^{2}-k_{o}^{2} \varepsilon_{r i}
$$

(3)

In Eq. (3) k0 denotes the free space wavenumber (k0 $=2 \mathrm{f0} / \mathrm{c}$ where $\mathrm{c}$ is the free-space celerity of light). In the dispersion equation Eq.(1) we can substitute $\beta$ by $\Pi /$ Land consequently, from resolution of the resulting resonant equation, derive the resonant frequency f0 of the first resonant mode. In particular, we can predict the resonant frequency when varying the air gap thickness h2 and then, estimate the frequency shift when applying a pressure on the silicon membrane (the membrane displacement is assumed to be uniform above the coplanar resonator). From resolution of the resonant equation with dimensions given in the caption of Figure 3 with $\mathrm{h} 1=1 \mathrm{~mm}$ and $\mathrm{h} 3=$ $0.4 \mathrm{~mm}$, a shift of $6.3 \mathrm{GHz}$ in the millimetre-wave resonant frequency of the coplanar resonator is numerically found when varying the air gap thickness $\mathrm{h} 2$ from 0.3 microns to 3 microns. This high-sensitivity of the resonant frequency to such small membrane deflection is the consequence of the rapid variation of the effective relative permittivity $\operatorname{\epsilon reff}=(\beta / \mathrm{k} 0) 2$ when varying the air gap thickness (see Figure 5). It results from a strong perturbation of the evanescent EM field in this region when approaching the silicon membrane interface to the transmission line. The proposed sensor is very sensitive to the air gap variation but not to the technological parameters: the different pro- cess steps are well-known (Pyrex etching, anodic bonding, and membrane realization). This process flow has proven its reliability in the fabrication of capacitive pressure sensors [15]. Fixed membrane sensors are used as reference sensors for the calibration step. Since the technological dispersion is limited, due to the well-known process, difficulties in sensor calibration are consequently minimized.

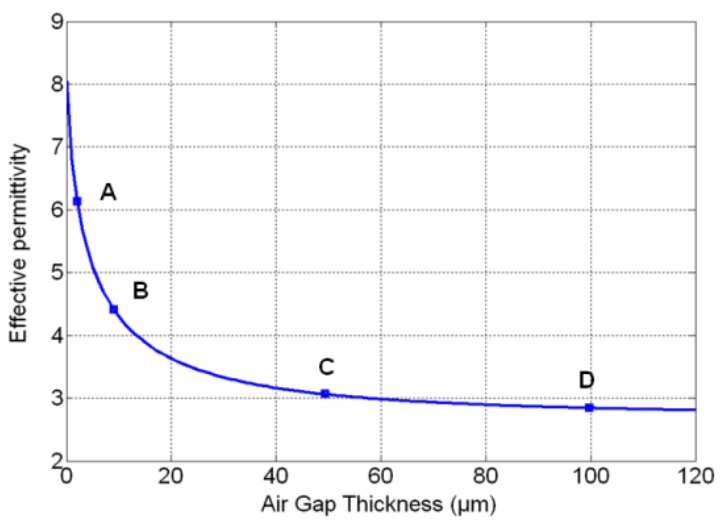

Fig. 5. Variation of the effective relative permittivity of the coplanar line shown in Figure 4 versus the air gap thickness h2 (i.e., the distance between the coplanar line and the silicon membrane interface)

In Figure 6, the computed magnitude of the electric field is displayed for various air gap thicknesses (see Figure 5 for the definition of $\mathrm{A}, \mathrm{B}, \mathrm{C}$ and $\mathrm{D}$ points). We observe stationary waves in the silicon membrane $(\mathrm{p} 2 \mathrm{n}$ is pure imaginary number for some low order n) and evanescent waves in the air gap region ( $\mathrm{p} 3 \mathrm{n}$ is real for any $\mathrm{n})$. Moreover when approaching the membrane to the coplanar line (or equivalently decreasing the air gap thickness) the electric field migrates gradually from the slots of the coplanar line to the air gap region and the electromagnetic coupling 
between the coplanar line and the membrane increases: more and more electric energy penetrates inside the dielectric membrane and the resulting relative effective permittivity increases (as found theoretically, see Figure 5) The first resonant frequency $c / \sqrt{\varepsilon_{r e f f}} L$ of the corresponding coplanar quarter-wave coupled-line resonator will then significantly decrease when approaching the membrane to the coplanar line from 3 micrometers to 0.3 micrometers. Since the effective permittivity of coplanar transmission line is found to be highly depending on the air gap thickness (or to the applied pressure), passive pressure sensor based on the proposed resonator is expected to have high sensitivity.
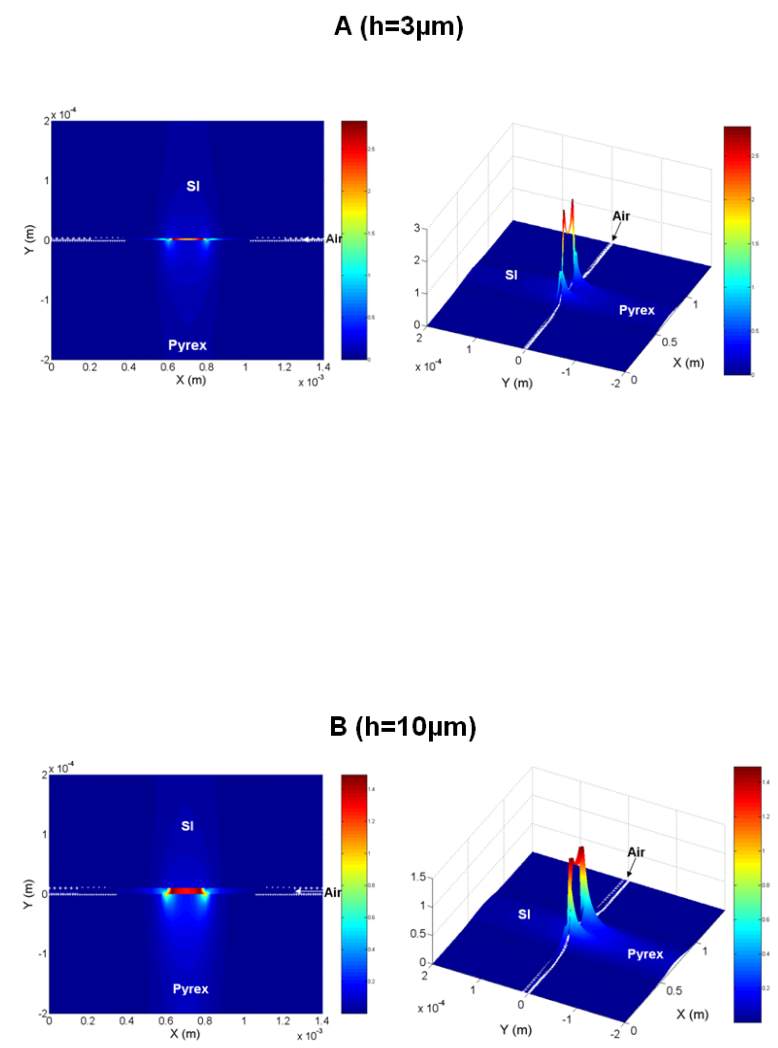
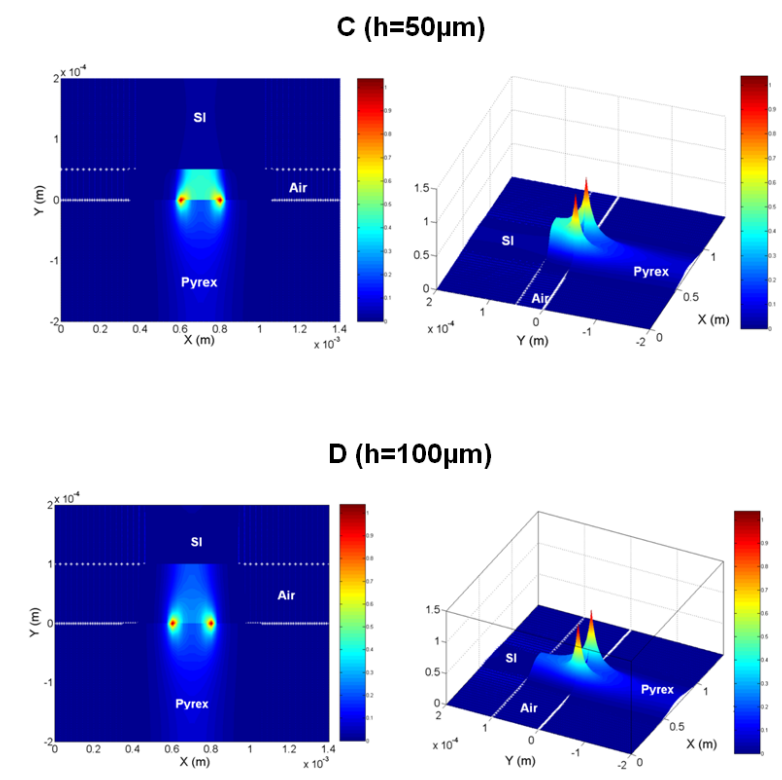

Fig. 6. Computed EM field distribution for different air gap thickness (for A, B, C and D points indicated in figure 5)

\section{Measurement results}

Pressure sensors with 50 microns thick silicon circular membrane (2800 microns diameter) have been fabricated. Measurement results under real working conditions, obtained from a specific RF/Pressure measurement bench, have been reported and have revealed a high sensitivity of $370 \mathrm{MHz} /$ bar in Ka-band. Detailed discussion concerning the electromagnetic characterization of the sensor in presented in previous work [16]. Figure 7 shows the spectral sensor response when no pressure is applied. The measured return loss reveals a resonant frequency fr $=39,46$ GHz with a magnitude of $-15,34 \mathrm{~dB}$. Previous work [16] presented a parametric study in which the relation between the membrane thickness and the resonant frequency is established. The resonant frequency of $39,46 \mathrm{GHz}$ is in adequacy with the membrane thickness of 50 microns. 


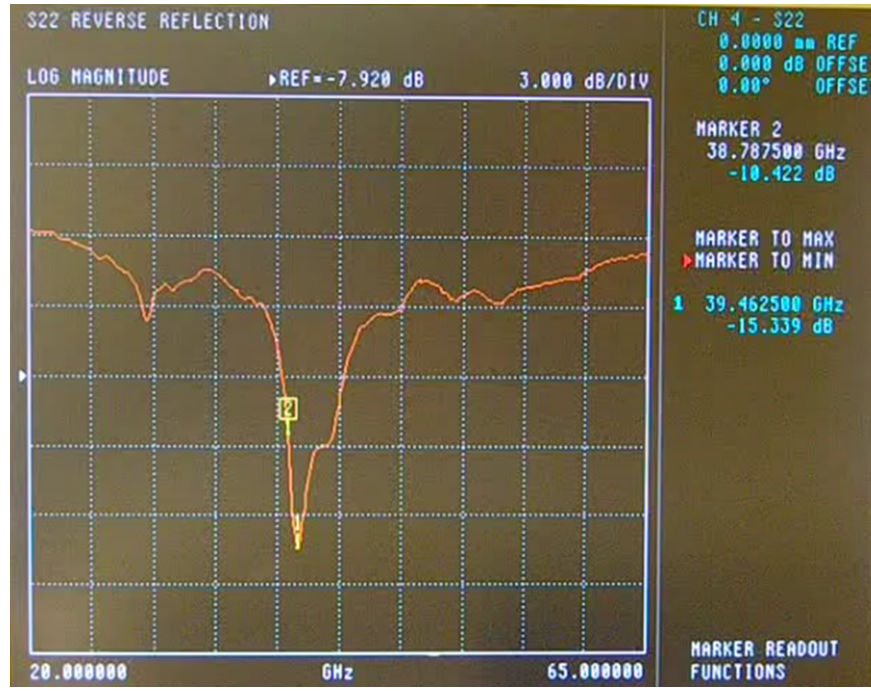

Fig. 7. Spectral sensor response when no pressure is applied on the membrane

Here new measurement results dealing with the wireless interrogation technique are presented. The Pressure Sensor is interrogated in indoor environment using FMCW radar reader. As shown in Fig.8 and Fig.9, the passive pressure sensor is connected to a broadband horn antenna via a coaxial (delay) line of 1 meter of length. The other port in loaded by $50 \Omega$ matched impedance. The sensor is viewed as load impedance depending on the applied pressure.

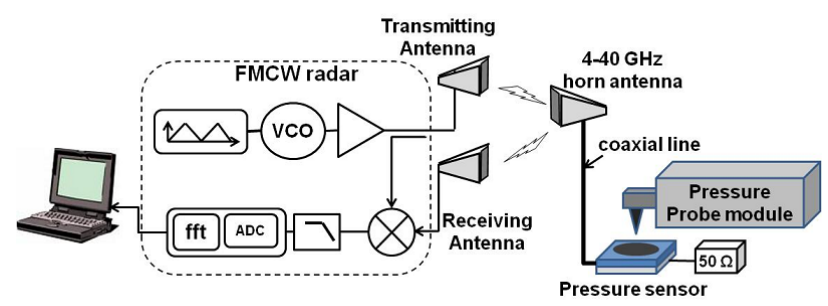

Fig. 8. Schematic principle of the FMCW Radar interrogation technique of the EM passive pressure sensor

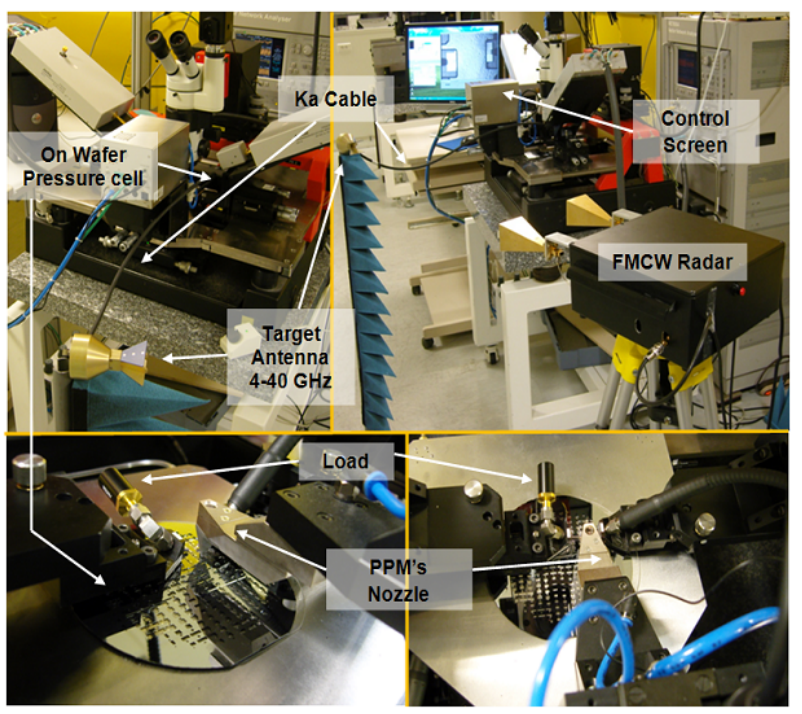

Fig. 9. Experimental set-up used for the applied pressure measurement using FMCW radar

The sawtooth FM-signal of the reader, in Ka band, is transmitted in direction of the sensor antenna and mixed with the backscattered signal to produce few ten $\mathrm{KHz}$ (beat frequency) radar signal. As expected, we have observed that the level of the backscattered signal changes in response to applied pressure. In particular, between 0 to 2.5 bars we obtained from Figure 10 a measured sensitivity close to $0.8 \mathrm{dBm} / \mathrm{Bar}$.

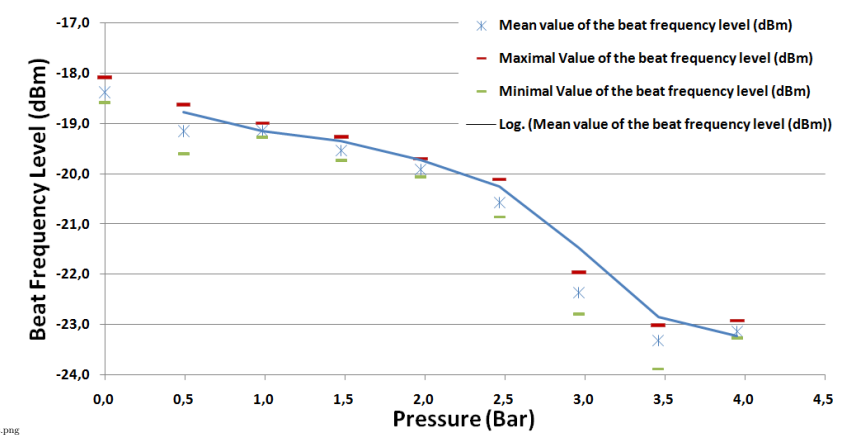

Fig. 10. Backscattered signal level detected by the radar versus the applied pressure on the passive sensor 
These measurements show that the proposed wireless system (composed of: passive sensor, antenna and FMCW radar) is functional and allows the remote measurement of the applied pressure with no need for signal processing circuits, power supplies or batteries to be integrated within the sensor units. The sensor unit is then viewed as a passive scatterer having a reflectivity (or radar cross section) that varies with -or is controlled by- the applied pressure.

\section{Conclusion}

In conclusion, this paper presents a detailed working overview Scherr, Ulrich Woland Frank Schmidt, IEEE transactions on of the EM pressure sensor and brings a proof of concept of an original remote pressure measurement technique using EM transduction passive sensor as electromagnetic scatterer: the applied pressure on the EM passive sensors modifies their radar echo magnitude. Using the FMCW radar interrogation method, the pressure can then be remotely measured. Experimental results presented here demonstrate the feasibility of the remote physical quantities measurement from the backscattered echo of EM passive sensors. For the first time, the functioning and so the feasibility of this passive wireless system (EM pressure sensor connected to the antenna) under real working condition is demonstrated. Using this wireless interrogation technique, sensitivity of $0.8 \mathrm{dBm} /$ bar is measured for the very first sensor prototype. Future work will be the optimization of the pressure measurement unit. Solutions for transportable version of the whole system are being studied. The optimized system will be used for the valida- tion of long interrogation range (Around thirty meters). Identification techniques, for the pressure cell identification among wireless sensor network, are also under investigation.

\section{References}

1. Sir Magdi Yacoub, Body Sensor Networks (Springer-Verlag London, 2006)

2. Elena Gaura and Robert Newman, Smart MEMS and Sensor Systems (Imperial College Press, 2006)

3. Leonhard Reindl, Gerd Scholl, Thomas Ostertag, Holger ultrasonics, ferroelectrics, and frequency control. 45, no. 5, (1998).

4. Alfred Pohl, IEEE transactions on ultrasonics, ferroelectrics, and frequency control. 47, no. 2, (2000).

5. Gernot Schimetta, Franz Dollinger, and Robert Weigel, IEEE Transactions on Microwave Theory and Techniques. 48, no. 12, (2000).

6. Jason D. Sternhagen, Cyle E. Wold, Wade A. Kempf, Matt Karlgaard, Kraig D. Mitzner, Russell D. Mileham, and David W. Galipeau, Sensors Journal, IEEE. 2, Issue 4, (2002) Page(s): $301-306$.

7. M.M.Jatlaoui, P.Pons, H.Aubert, in Proceedings of the 37th European Microwave Conference (Munich, 2007) pp.736-739 8. M.M.Jatlaoui, P.Pons, H.Aubert, in Proceedings of the IEEE International Microwave Symposium (Atlanta, Georgia, USA, 2008)

9. M.M.Jatlaoui, F.Chebila, P.Pons, H.Aubert, in Proceedings of the 39th European Microwave Conference (Rome, Italy, 2009) pp.53-56 
10. G. L. Matthei, L. Young, and E. M. T. Jones, Microwave Filters,Impedance Matching Networks, and Coupling Structures( Norwell,MA: Artech House, 1980)

11. Ingo Wolff, Coplanar Microwave Integrated Circuits( Wiley, 2006)

12. H. Aubert, B. Souny, H. Baudrand, IEEE Transactions on Microwave Theory and Techniques. vol. MTT-41, (1993) pp. $450-456$

13. F.Bouzidi, H. Aubert, D. Bajon, H. Baudrand, IEEE Transactions on Microwave Theory and Techniques. vol. MTT-45, (1997) pp.869-876

14. H. Aubert, H. Baudrand, Electromagnetism by equivalent circuits (in french) (Cpadus-Editions, Collection Polytech, 2003)

15. G. Blasquez, C. Douziech, P. Pons, Sensors and Actuators A: Physical. vol. 93, Issue 1, (2001) pp.44-47

16. M.M.Jatlaoui, F.Chebila, I.Gmati, P.Pons, H.Aubert, in Proceedings of the Transducers 2009, 15th International Conference on Solid-State Sensors, Actuators and Microsystems (Denver, Colorado, USA, 2009) Page (s):1742-1745 